Depth profiling of the modification induced by high-flux deuterium plasma in tungsten and tungsten-tantalum alloys

This content has been downloaded from IOPscience. Please scroll down to see the full text. 2014 Nucl. Fusion 54123013

(http://iopscience.iop.org/0029-5515/54/12/123013)

View the table of contents for this issue, or go to the journal homepage for more

Download details:

IP Address: 157.193.64.157

This content was downloaded on 09/03/2015 at 10:58

Please note that terms and conditions apply. 


\title{
Depth profiling of the modification induced by high-flux deuterium plasma in tungsten and tungsten-tantalum alloys
}

\author{
Y. Zayachuk ${ }^{1,2,3,4}$, A. Manhard ${ }^{5}$, M.H.J. ‘t Hoen ${ }^{6}$, W. Jacob ${ }^{5}$, \\ P.A. Zeijlmans van Emmichoven ${ }^{6,7}$ and G. van Oost ${ }^{2}$ \\ ${ }^{1}$ SCK•CEN, Trilateral Euregio Cluster, Boeretang 200, 2400 Mol, Belgium \\ ${ }^{2}$ Department of Applied Physics, Ghent University, St Pietersnieuwstraat 41, 9000 Ghent, \\ Belgium \\ ${ }^{3}$ Department of Materials, University of Oxford, Parks Road, Oxford OX1 3PH, UK \\ ${ }^{4}$ Culham Centre for Fusion Energy, Culham Science Centre, Abingdon OX14 3DB, UK \\ ${ }^{5}$ Max Planck Institute for Plasma Physics, Boltzmannstrasse 2, 85748 Garching, Germany \\ ${ }^{6}$ FOM Institute DIFFER, Trilateral Euregio Cluster, Edisonbaan 14, 3439 MN Nieuwegein, \\ The Netherlands \\ ${ }^{7}$ Aviation Academy, Amsterdam University of Applied Sciences, Weesperzijde 190, 1097 \\ DZ Amsterdam, The Netherlands
}

E-mail: yevhen.zayachuk@materials.ox.ac.uk

Received 17 July 2014, revised 2 October 2014

Accepted for publication 22 October 2014

Published 13 November 2014

\begin{abstract}
The present work reports the results of an experimental study of the depth distribution and fluence dependence of deuterium plasma-induced material modification of tungsten and tungsten-tantalum alloys. Plasma-induced damage was created by exposure to high-flux deuterium plasma in the plasma generator Pilot-PSI, followed by the degassing and subsequent decoration of created defects with deuterium by another plasma exposure. The depth distribution of deuterium from the decorating exposure reflects the distribution of plasma-induced defects. Depth profiling of this decorating deuterium, was performed by nuclear reaction analysis. It was found that plasma-induced material modification, which manifested itself as an increase of the deuterium concentration in the samples pre-exposed with high-flux plasma in comparison to the samples without such pre-exposure extends down to more than $5 \mu \mathrm{m}$ from the surface. This increase features a tendency to saturation with increasing fluence of the damaging high-flux plasma. Over the entire probing range, with the exception of the narrow surface region and the deep region beyond $5 \mu \mathrm{m}$, the deuterium content is lower in pre-exposed W-Ta than in similarly pre-exposed W. Sub-surface features formed as a result of high-flux plasma exposure were studied with the help of focused ion beam cross-sectioning. W was found to contain plasma-induced cavities down to much larger depth than W-Ta.
\end{abstract}

Keywords: deuterium retention, linear plasma generator Pilot-PSI, tungsten, tungsten-tantalum

(Some figures may appear in colour only in the online journal)

\section{Introduction}

It has been repeatedly reported that exposure of tungsten-based materials to deuterium plasma can lead to modification of these materials. It can manifest itself by the formation of sub-surface cavities associated with surface blisters [1,2], as well as by the generation of crystallographic defects [3]. Plasma-induced material modification was also found to significantly influence deuterium retention, leading to the emergence of a history effect, i.e. the enhancement of the deuterium retention after the pre-exposure to low-energy, high-flux deuterium plasma [4]. What has not been addressed up to now is how deep into the material the region influenced by the plasma-induced modification reaches, and how this modification evolves with the exposure fluence.

Tungsten is known to have certain unfavourable properties hindering its use as a plasma-facing material (PFM), such as susceptibility to surface cracking under ELM-like transient heat loads [5] due to the high brittle-to-ductile transition temperature [6]. Several alloys were proposed with advantages in the sense of thermo-mechanical properties, one of such alloys being W-Ta [7]. This alloy was demonstrated to have better resistance to degradation under repetitive ELM-like heat loads [8]. It was also demonstrated to be affected by plasma exposure in a manner similar to $\mathrm{W}$, i.e. it features a similar history effect [4]. 
In this contribution we present the results of an experiment to determine the depth distribution of plasma-induced material modification in tungsten and its evolution with fluence. Since the assessment of W-Ta as a candidate PFM is in progress [4, 9-12], we studied the depth distribution of plasma-induced material modification for W-Ta as well.

\section{Experiment}

In order to study the depth distribution of plasma-induced material modification in $\mathrm{W}$ and $\mathrm{W}-\mathrm{Ta}$ alloy, samples of both materials were exposed to a high-flux deuterium plasma and analysed with a complementary set of analysis techniques including nuclear reaction analysis (NRA), focused ion beam (FIB) cutting and scanning electron microscopy (SEM).

Forged powder metallurgical W, as well as a W-Ta alloy containing 5 mass $\%$ of Ta, were investigated. The materials were provided by Plansee. All investigated polycrystalline samples were mechanically polished to mirror finish with $0.05 \mu \mathrm{m}$ alumina polishing suspension, and then annealed in vacuum at $1300 \mathrm{~K}$ for $1 \mathrm{~h}$. During annealing the samples were not recrystallized. The samples were discs with a diameter of $20 \mathrm{~mm}$ and a thickness of $1 \mathrm{~mm}$.

Plasma exposures were performed in the linear plasma generator Pilot-PSI (FOM Institute DIFFER [13]). In this device the plasma is generated by a cascaded arc discharge. Electron temperature and density profiles (and therefore also particle and heat flux profiles) within the plasma beam are roughly Gaussian with a FWHM of $\sim 1 \mathrm{~cm}$. During the plasma exposure, the sample is fixed to the cooling plate by a clamping ring.

The ion flux arriving at the surface of the specimen is calculated from the electron temperature and density measured by Thomson scattering. The surface temperature of each specimen is monitored by an IR camera.

Pilot-PSI operates in a pulsed regime, which means that in order to accumulate high ion fluences it is necessary to perform several sequential exposures. Each of these exposures (referred to in the following as 'reference shots') was performed under identical conditions, with the maximum D ion flux of $5-8 \times 10^{23} \mathrm{~m}^{-2} \mathrm{~s}^{-1}$, time duration of $70 \mathrm{~s}$, ion fluence (calculated in the location of maximum ion flux) of $\sim 5 \times 10^{25} \mathrm{~m}^{-2}$, maximum surface temperature of $\sim 450 \mathrm{~K}$ and ion energy (determined by the sample bias voltage) of $50 \mathrm{eV}$.

For the deuterium depth profiling we implemented the commonly used $\mathrm{D}\left({ }^{3} \mathrm{He}, \mathrm{p}\right) \alpha$ reaction. The use of this reaction for the investigation of $\mathrm{D}$ in tungsten is extensively described in [14]. Depth profiling was performed at a specific point corresponding to the centre of the plasma beam where the ion flux is highest (determined during the exposure by the IR camera as the location with the highest surface temperature) for each individual sample. The energies used for the detailed depth profiling were $500 \mathrm{keV}, 690 \mathrm{keV}, 1.2 \mathrm{MeV}, 1.8 \mathrm{MeV}$, 2.4 MeV, 3.2 MeV and 4.5 MeV.

The interpretation of raw NRA data is complex. The reason is mainly that the particular D- ${ }^{3} \mathrm{He}$ reaction crosssection is broad yielding a large uncertainty in depth from which the detected protons are coming. Thus, the conversion of raw NRA data (alpha and proton energy spectra) into depth profiles requires a complex numerical analysis involving forward simulations, which were performed with the use of the software packages SimNRA [15] and NRADC [16].

FIB cross-sectioning and in situ imaging of the cuts were performed using a HELIOS NanoLab 600 (FEI) workstation. Details of the FIB procedure can be found in [17].

The experiment aimed at two major points: determination of depth distribution and fluence dependence of the plasma-induced material modification in tungsten; and (ii) comparison of plasma-generated damage in tungsten and tungsten-tantalum alloy. For the first goal a set of W samples was exposed to several different D fluences. These exposures are referred to as 'damaging' exposures. The damaging fluences were chosen as 1 and 20 reference shots, corresponding to the fluences of $\sim 5 \times 10^{25}$ and $10^{27} \mathrm{~m}^{-2}$. For the second goal, a W-Ta sample was exposed to the highest damaging fluence of 20 reference shots, and later used for comparison with the analogous $\mathrm{W}$ sample. After the damaging, deuterium was removed from each sample by heating it to $1300 \mathrm{~K}$ and holding it at this temperature for $5 \mathrm{~min}$ (in our earlier experiments performed under similar conditions (e.g. [9]) it was shown that this is indeed sufficient to remove practically all retained deuterium). After that, all samples with pre-exposure history, as well as the one without such history (the reference sample), were exposed again, this time to a single reference shot of $\sim 5 \times 10^{25} \mathrm{~m}^{-2}$ each. These exposures are referred to as 'probing' ones.

The concept of the experiment was the following: during the damaging exposures certain levels of material modification - that is, certain distributions of plasma-induced defects, or more broadly, distributions of trapping capacity (as different types of defects might be able to trap deferent amount of deuterium)-are created in the samples. A subsequent TDS run removes the deuterium that was trapped during the damaging exposure from these defects, ideally leaving defects themselves intact. This was shown by earlier observations of the history effect [4] proving that indeed material modifications induced by the high-flux damaging exposure at least partly survive the heat treatment (which includes heating to $1300 \mathrm{~K}$, albeit for a short time of $5 \mathrm{~min}$ only) during the TDS measurement. During the probing exposures these defects are again decorated with deuterium. NRA measurements after the probing exposure visualize the depth distribution of the retained deuterium, which in turn follows the depth distribution of the defects generated during the damaging exposure.

SEM imaging of FIB cross-sections of the surfaces of these samples directly yields the depth distribution of macroscopic cavity-type defects. From this, one can get direct information about the correlation between the distributions of macroscopic plasma-induced defects and deuterium trapped on them.

\section{Experimental results}

\subsection{Nuclear reaction analysis}

Figure 1 presents the results of NRA deuterium depth profiling performed for all the samples at the position corresponding to the centre of the probing beam. We consider the measured deuterium depth profiles to be also representations of depth profiles of concentrations of plasma-induced defects. 


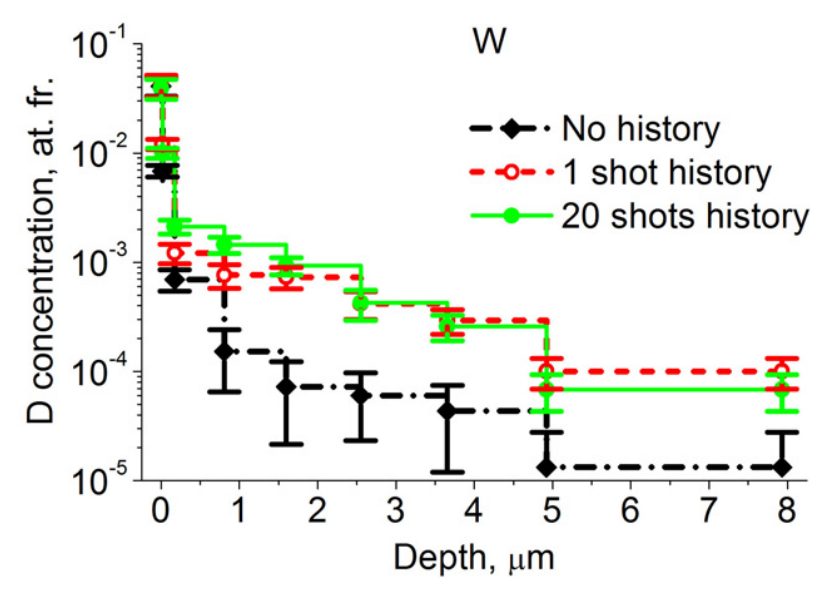

Figure 1. D depth profiles in $\mathrm{W}$ samples with different damaging histories.

Several characteristic features can be seen from the comparison of the depth distributions for the W samples with different pre-exposure histories (figure 1): (i) the exposure history practically doesn't influence the surface concentration of traps. By 'surface' here the first $16 \mathrm{~nm}$ from the surface is meant; $16 \mathrm{~nm}$ constitutes the best achievable depth resolution of the NRA measurement at the minimum probing energy of $500 \mathrm{keV}$ (when both proton and alpha particles energy spectra are taken into account). It is quite similar for all the samples, independently of their history, and close to 4 at $\%$ averaged over these first $16 \mathrm{~nm}$. (ii) The exposure history strongly influences the concentrations of traps in the volume of the samples below the immediate surface region discussed above. This concentration is higher for the samples with damaging history than for the sample without such history over the entire probed range of $8 \mu \mathrm{m}$. In this respect it should be noted that the deepest layer is necessarily quite broad, reflecting the decrease of the NRA depth resolution at large depth. It is clear that the deuterium content beyond $5 \mu \mathrm{m}$ is higher in the samples with history as compared to the sample without history, but it is impossible to say whether the difference is present within the entire range of $5-8 \mu \mathrm{m}$. In any case, we are certain that the increase of deuterium concentration as a result of plasma pre-exposure extends beyond $5 \mu \mathrm{m}$. (iii) The main changes of the distribution of traps occur during the early period of the exposure history. The difference between samples without history and the one with a single history shot is much larger than between samples that underwent 1 and 20 shots of damaging exposures. In the sample with 20 shots history, the concentration of traps is somewhat higher than in the sample with 1 shot history, mostly in the region from $\sim 200 \mathrm{~nm}$ to $\sim 1.5 \mu \mathrm{m}$, outside of which it is almost identical for the two samples.

Figure 2 presents the comparison between the depth distributions reconstructed for $\mathrm{W}$ and $\mathrm{W}-\mathrm{Ta}$ samples with the longest pre-exposure histories of 20 shots. Again, the surface concentration (within first $16 \mathrm{~nm}$ ) is essentially identical for $\mathrm{W}$ and W-Ta samples, being close to $4 \mathrm{at} \%$. However, the depth distribution of traps is significantly different for $\mathrm{W}$ and $\mathrm{W}-\mathrm{Ta}$. The main difference is in the sub-surface region (first $\sim 200 \mathrm{~nm}$ from the surface). The concentration of traps in $\mathrm{W}$ decreases rather smoothly with depth. On the other hand, in

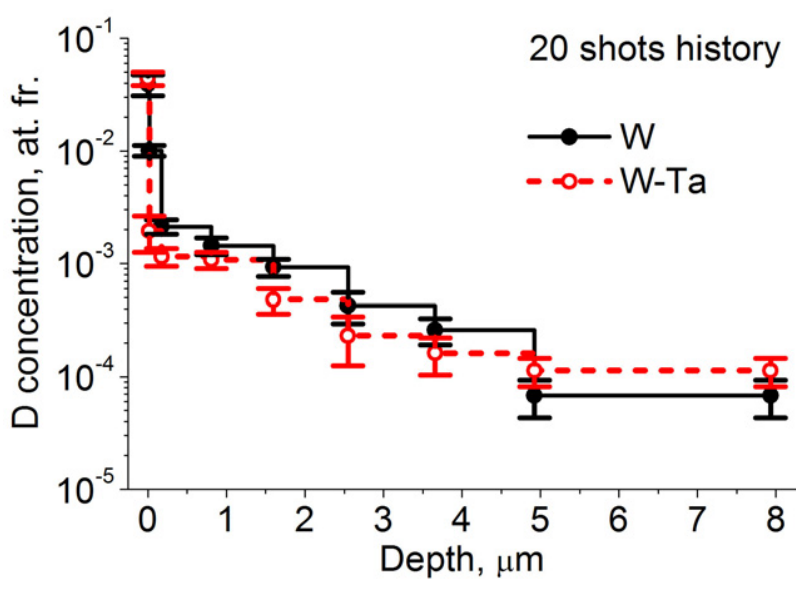

Figure 2. D depth profiles in $\mathrm{W}$ and $\mathrm{W}-$ Ta samples with 20 shots history.

W-Ta this concentration drops down abruptly in the thin subsurface layer, and only then starts decreasing smoothly. The concentration of traps is higher in $\mathrm{W}$ as compared to $\mathrm{W}-\mathrm{Ta}$ down to $\sim 5 \mu \mathrm{m}$.

In the deepest probed range between 5 and $8 \mu \mathrm{m}$ the deuterium content is somewhat higher in W-Ta than in W. Similar to the case of comparing W samples with different pre-exposure histories, it is impossible to say how deuterium is distributed exactly in this deep region. However, it is certain that the region where deuterium concentration in $\mathrm{W}$ is higher than in W-Ta extends at least down to $5 \mu \mathrm{m}$.

\subsection{FIB cross-sectioning}

High-flux exposures like the ones used here for both damaging and probing exposures lead to noticeable blistering of the surfaces of both W and W-5\% Ta [11]. In order to determine the structure of the sub-surface cavities corresponding to these blisters, and to correlate the appearance of these cavities to the observed deuterium depth distributions, FIB cross-section cuts were performed.

Images from a cross-section cut of the $\mathrm{W}$ sample with 20 shots history are shown in figures $3(a)$ and $(b)$. One can see that large plateau-like surface structures are caused by sub-surface cavities created by intergranular cracks, so that the blister cap is formed by entire sub-grains being pushed out of the surface. Such cavities are located at depths of $\sim 1 \mu \mathrm{m}$ from the surface. Other cavities causing the emergence of surface structures are intragranular cracks located very close the surface, i.e. within a range of tens to hundreds of nanometres. The general trend is that small surface blisters correspond to sub-surface cavities located close to the surface, while the cavities leading to the emergence of larger blisters are located deeper in the material. It should be noted that all the cavities that lead to observable surface structures are located within the $\sim 1.5 \mu \mathrm{m}$ depth range. In addition, FIB cuts reveal the existence of intergranular cracks located farther from the surface-down to $\sim 5 \mu \mathrm{m}$ deep. These, however, do not lead to clearly visible surface structures and therefore are not identifiable by surface SEM imaging alone.

A similar cut of the surface of the W-Ta sample is shown in figure 4. The major difference with the corresponding $\mathrm{W}$ 

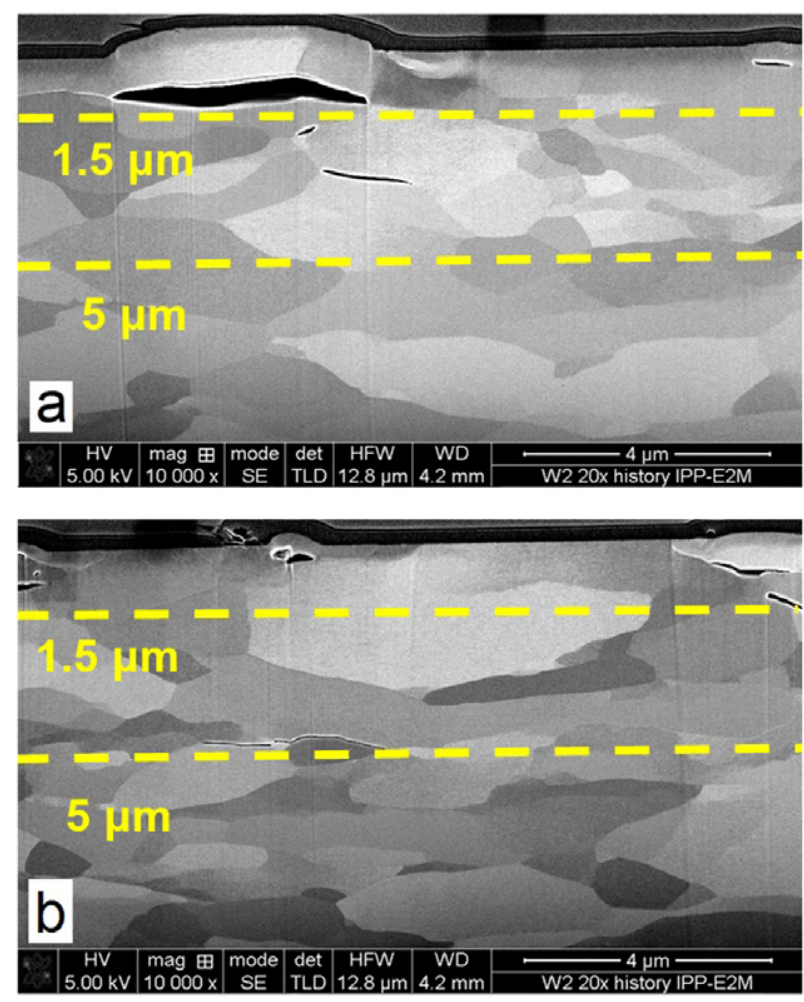

Figure 3. Cross-sectional images of W sample with 20 shots history, at two different locations. The dark layer on top is an amorphous Pt: C layer that protects the sample surface during FIB milling. Depth scale is corrected for the cross-section being viewed under an angle of $38^{\circ}$.

sample is the fact that all the observed sub-surface cavities are located close to the surface. No cavities are observed in $\mathrm{W}-\mathrm{Ta}$ at large depths, i.e. beyond $\sim 1.5 \mu \mathrm{m}$. In addition it should be kept in mind that the amount of blisters observed by surface SEM imaging on W-Ta is much smaller than on similarly exposed W [11].

\section{Discussion}

The major experimental findings of this work can be summarized as follows: (i) the region of enhanced trapping as a result of high-flux deuterium plasma exposure extends down to more than $5 \mu \mathrm{m}$; (ii) the concentration of plasmainduced traps exhibits a tendency towards saturation; (iii) the concentration of traps within the plasma-affected region in preexposed $\mathrm{W}$ is higher than that in similarly pre-exposed $\mathrm{W}-\mathrm{Ta}$ under the conditions investigated here.

In order to better visualize which depth ranges predominantly contribute to the differences in total deuterium inventories, we present the depth distribution of the total amounts for different characteristic depth ranges. We define these ranges in the following way: (i) the 'surface' region from 0 to $16 \mathrm{~nm}$ from the sample surface roughly covers the implantation range as determined by SRIM modeling [18]; as mentioned above, $16 \mathrm{~nm}$ constitute the NRA depth resolution at the surface for the minimum probing energy of $500 \mathrm{keV}$; (ii) the 'sub-surface' between $16 \mathrm{~nm}$ and $1.5 \mu \mathrm{m}$ is defined in correlation with the location of cavities observed in FIB crosssections; this is the region where the cavities that lead to the

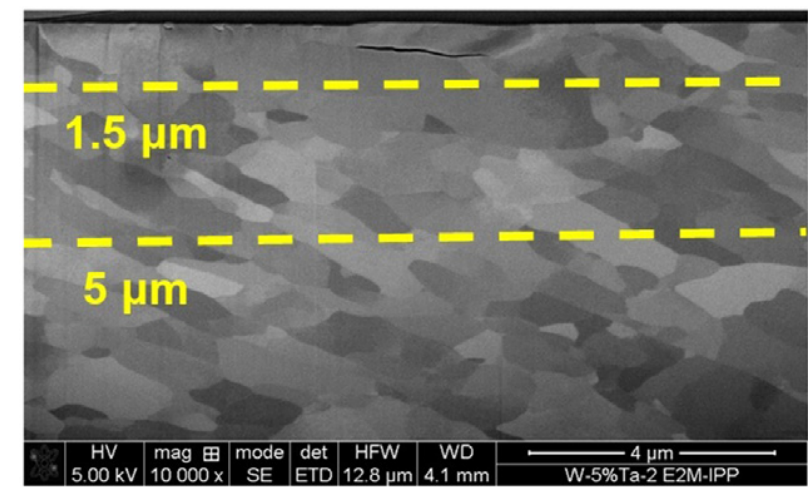

Figure 4. Cross-sectional image of W-Ta sample with 20 shots history. The dark layer on top is an amorphous $\mathrm{Pt}: \mathrm{C}$ layer that protects the sample surface during FIB milling. Depth scale is corrected for the cross-section being viewed under an angle of $38^{\circ}$.

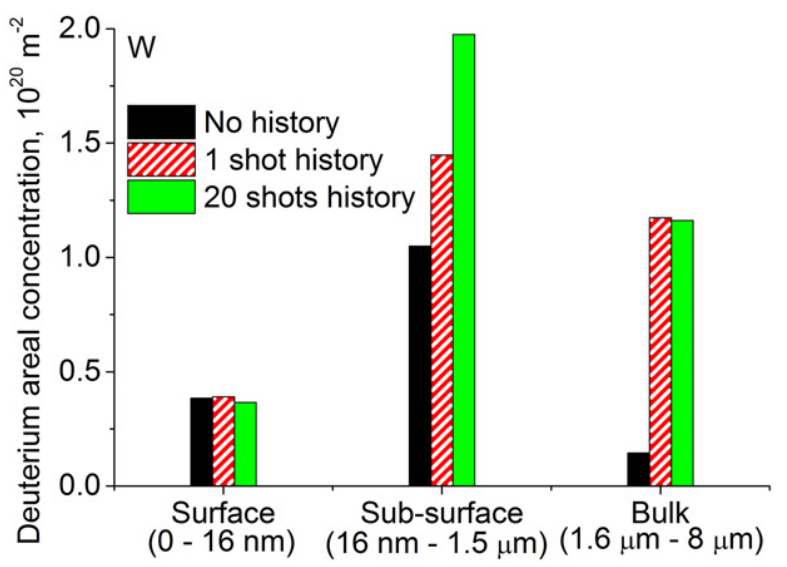

Figure 5. Distributions of deuterium inventories between characteristic depth ranges for W samples with different histories.

emergence of surface structures are located; (iii) the 'bulk' between 1.5 and $8 \mu \mathrm{m}$ spans down to the end of the NRA probing range.

Figure 5 presents comparison of such distributions for the $\mathrm{W}$ samples with different damaging histories. For all the investigated samples the deuterium amount in the 'surface' region is essentially identical. Since this region includes the implantation range, this is the region that is most immediately affected by the ion implantation. It is interesting to note that this inventory does not seem to be influenced by the exposure pre-history. Moreover, it is not affected by the presence of $\mathrm{Ta}$ either.

For the $\mathrm{W}$ sample without history, the vast majority of the detected deuterium is retained within the first $\sim 1.5 \mu \mathrm{m}-$ that is, within surface and sub-surface regions. Only a small fraction (approximately 9\%) is retained in the bulk beyond $1.5 \mu \mathrm{m}$ from the surface. On the other hand, in the pre-exposed samples the contribution of the bulk to the total retention is much higher (between $30 \%$ and $40 \%$ ). This difference in bulk retention for the pre-exposed and not pre-exposed samples indicates that the plasma-induced material modification is strong enough to significantly influence the retention, as it was earlier demonstrated by the observation of the history effect of retention due to high-flux D plasma exposure [4]. Moreover, it also clearly indicates that enhancement of retention as a 


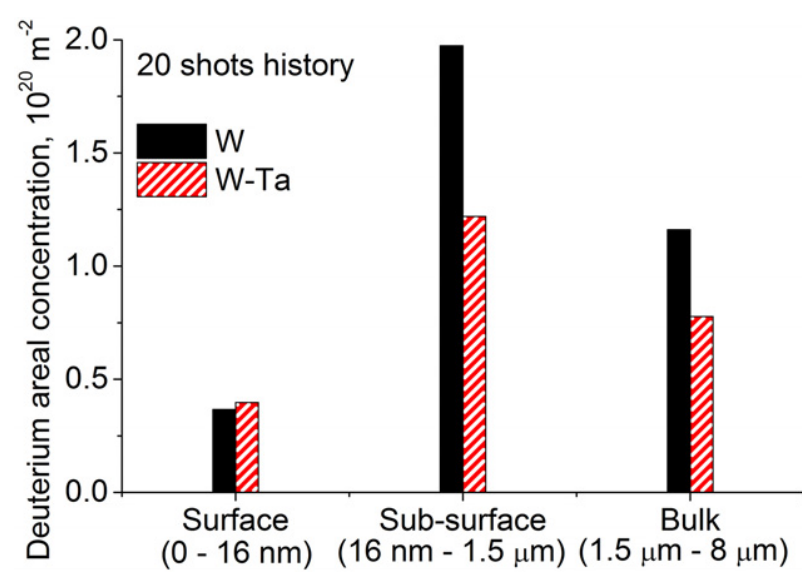

Figure 6. Distributions of deuterium inventories between characteristic depth ranges for W and W-Ta samples with 20 shots history.

result of high-flux plasma exposure occurs not only in the implantation zone, but at depths down to several micrometres.

Figure 6 presents comparison of distributions in characteristic depth ranges for $\mathrm{W}$ and $\mathrm{W}-\mathrm{Ta}$ samples with the damaging history of 20 shots. In the pre-exposed W-Ta also a significant fraction of deuterium content is retained in the bulk $(\sim 32 \%)$. Both in the sub-surface and in the bulk, the deuterium inventories in pre-exposed $\mathrm{W}-\mathrm{Ta}$ are lower than that in the similarly pre-exposed $\mathrm{W}$.

A correlation can be established between the observed depth distribution of the traps and that of the sub-surface cavities imaged by FIB. The sub-surface region, in both W and $\mathrm{W}-\mathrm{Ta}$, is the one where the sub-surface cavities which are visible as surface blisters are located. However, in the bulk region the character of macroscopic plasma-induced modifications strongly differ in $\mathrm{W}$ and $\mathrm{W}-\mathrm{Ta}$. In particular, the range between $\sim 1.5$ and $\sim 5 \mu \mathrm{m}$ contains intergranular cracks in $\mathrm{W}$ sample, but no such cracks in W-Ta sample. On the other hand, beyond $5 \mu \mathrm{m}$ neither material shows evidence of intergranular cracking. Comparing this to the deuterium depth distributions in both materials it can be seen that the depth range between the surface and $\sim 5 \mu \mathrm{m}$, where deuterium content is higher in $\mathrm{W}$ as compared to $\mathrm{W}-\mathrm{Ta}$, is simultaneously the range where $\mathrm{W}$ sample features multiple intergranular cracks-more numerous, it should be emphasized, than in W-Ta. On the other hand, in the depth range beyond $\sim 5 \mu \mathrm{m}$ no cracks are observed in any of two materials, and in this depth range deuterium inventory in $\mathrm{W}-\mathrm{Ta}$ exceeds that in $\mathrm{W}$.

These observations indicate that in W and in W-Ta the bulk trapping mechanisms might be different: in $\mathrm{W}$, macroscopic cavities seem to significantly contribute to the total retention, possibly in the form of gaseous deuterium contained within them, while this is not the case in W-Ta. Trapping on lattice defects can be suggested as a predominant trapping mechanism in the alloy.

To conclude the discussion we will compare the presented results with the recently published data on retention in $\mathrm{W}$ and $\mathrm{W}-5 \%$ Ta exposed to high fluences of high-flux plasma [11]. There it was demonstrated that under high-flux exposure the fluence dependence of deuterium retention has a tendency to saturation. The results presented here clearly indicate that under the condition of high-flux exposure deuterium retention occurs mainly on the plasma-generated trapping sites, and that the generation of these traps tends to saturation with the increase of high-flux plasma fluence. This supports the interpretation proposed in [11] that the saturation of the retention with the increase of fluence is indeed caused by the saturation of the amount of plasma-induced trapping sites.

\section{Conclusions}

An experiment on direct depth profiling of the plasma-induced material modification has been performed. In this experiment a set of W and W-Ta samples was exposed to different fluences $\left(\sim 5 \times 10^{25}-10^{27} \mathrm{~m}^{-2}\right)$ of high-flux $\left(\sim 10^{24} \mathrm{~m}^{-2} \mathrm{~s}^{-1}\right)$ deuterium plasma. The defects created during this treatment where then decorated with one further high-flux plasma discharge at a fluence of $\sim 5 \times 10^{25} \mathrm{~m}^{-2}$. The depth distribution of this deuterium was then probed by NRA. This deuterium depth distribution mirrors the depth distribution of the highflux plasma-induced material modifications. Macroscopic modifications (sub-surface cavities) were studied using FIB cross-sectioning.

It was found that plasma-induced material modificationthat is, generation of plasma-induced trapping sites-is not confined to the shallow sub-surface region around the implantation range. Significant modification in tungsten and tungsten-tantalum alloy occurs down to a depth of more than $5 \mu \mathrm{m}$. In tungsten, strong modification occurs already after the exposure to the lowest damaging fluence of $\sim 5 \times 10^{25} \mathrm{~m}^{-2}$ (one single reference plasma shot). The concentration of plasma-induced traps seems to tend towards saturation, as the difference between samples with damaging fluences of $\sim 5 \times 10^{25} \mathrm{~m}^{-2}$ and $10^{27} \mathrm{~m}^{-2}$ is relatively minor.

The concentration of traps in the $\mathrm{W}-\mathrm{Ta}$ sample preexposed to the highest damaging fluence of $10^{27} \mathrm{~m}^{-2}$ is noticeably smaller than that in the $\mathrm{W}$ sample exposed to identical damaging fluence down to at least $5 \mu \mathrm{m}$, with the exception of the narrow surface region.

The deuterium inventory in the surface region (first $\sim 16 \mathrm{~nm}$ from the surface) is independent of the pre-exposure history and of the presence of Ta.

Both $\mathrm{W}$ and $\mathrm{W}-\mathrm{Ta}$ were found to contain sub-surface cavities down to $\sim 1.5 \mu \mathrm{m}$ underneath the surface, leading to the emergence of surface blisters. In addition, $\mathrm{W}$ was found to contain cavities at larger depth, down to $\sim 5 \mu \mathrm{m}$, which do not correspond to any observable surface features. In W-Ta such deep cavities were not observed.

\section{Acknowledgments}

This work, supported by the European Communities under the contract of Associations between EURATOM and SCK•CEN, was carried out within the framework of the European Fusion Development Agreement. The views and opinions expressed herein do not necessarily reflect those of the European Commission. The authors would like to express gratitude to J. Dorner, M. Fusseder, Dr Th. Schwarz-Selinger and Dr M. Balden (Max Planck IPP) as well as to Dr V. Dubinko 
(Kharkiv Institute of Physics and Technology). Dr Dubinko would like to acknowledge the financial support of Erasmus Mundus FUSION-EP program.

\section{References}

[1] Wang W., Roth J., Lindig S. and Wu C.H. 2001 J. Nucl. Mater. 299 124-31

[2] Lindig S., Balden M., Alimov V.Kh., Yamanishi T., Shu W.M. and Roth J. 2009 Phys. Scr. T138 014040

[3] Ogorodnikova O.V., Roth J. and Mayer M. 2003 J. Nucl. Mater. 313469

[4] Zayachuk Y., 't Hoen M.H.J., Zeijlmans van Emmichoven P.A., Uytdenhouwen I. and van Oost G. 2012 Nucl. Fusion 52103021

[5] Hirai T., Pintsuk G., Linke J. and Batilliot M. 2009 J. Nucl. Mater. 390-391 751-4

[6] Gumbsch P. 2003 J. Nucl. Mater. 323 304-12

[7] Linke J. et al 2011 Nucl. Fusion 51073017

[8] Wirtz M., Linke J., Pintsuk G., Singheiser L. and Uytdenhouwen I. 2011 Phys. Scr. T145 014058
[9] Zayachuk Y., Bousselin G., Schuurmans J., Gasparyan Yu., Uytdenhouwen I. and Van Oost G. 2011 Fusion Eng. Des. 86 1153-6

[10] Zayachuk Y., 't Hoen M.H.J., Uytdenhouwen I. and Van Oost G. 2011 Phys. Scr. T145 014041

[11] Zayachuk Y., 't Hoen M.H.J., Zeijlmans van Emmichoven P.A., Terentyev D., Uytdenhouwen I. and van Oost G. 2013 Nucl. Fusion 53013013

[12] Schmid K., Rieger V. and Manhard A. 2012 J. Nucl. Mater. $426247-53$

[13] van Rooij G.J. et al 2007 Appl. Phys. Lett. 90121501

[14] Alimov V.Kh., Mayer M. and Roth J. 2005 Nucl. Instrum. Methods Phys. Res. B 234 169-75

[15] Mayer M. 1997 SimNRA User Guide, Report IPP 9/113, Max-Planck-Institut für Plasmaphysik, Garching, Germany

[16] Schmid K. and von Toussaint U. 2012 Nucl. Instrum. Methods Phys. Res. B 281 64-71

[17] Lindig S., Balden M., Alimov V.Kh., Manhard A., Höschen C., Höschen T., Tyburska-Püschel B. and Roth J. 2011 Phys. Scr. T145 014039

[18] Ziegler J.F., Ziegler M.D. and Biersack J.P. 2010 Nucl. Instrum. Methods Phys. Res. B 268 1818-23 